\section{Let's not fool ourselves. In RA, the ACR/EULAR remission criteria are not perfect!}

We were interested to read Dr Boer's recent eLetter, ${ }^{1}$ in which he outlines the merits of the American College of Rheumatology (ACR) and European League Against Rheumatism (EULAR) 2011 consensus remission criteria in rheumatoid arthritis (RA), ${ }^{2}$ and proposes that this definition equates with absence of disease.

The 2011 ACR/EULAR remission criteria do indeed hold several benefits over composite index-based remission definitions such as the disease activity score in 28 joints (DAS28). The ACR/EULAR criteria are widely regarded to be more stringent at defining remission than DAS28-erythrocyte sedimentation rate $(\mathrm{ESR})<2.6$, supported by a stronger correlation with lower rates of radiographic progression in the ACR/EULAR definition. ${ }^{2}$ Furthermore, the ACR/EULAR criteria were developed by consensus agreement among a panel of international RA experts with the express aim of defining remission, whereas DAS28 was developed with the primary intention of measuring disease activity for the purposes of treatment escalation. Although representing a significant international advance in defining remission, it is nevertheless important to acknowledge the several limitations inherent to the ACR/EULAR remission criteria.

First, the ACR/EULAR criteria are based on 28 joint counts that exclude important joint areas; for example, the feet-this shortcoming is described in the original ACR/EULAR criteria publication. Second, the ACR/EULAR Boolean criteria place a strict threshold on patient global assessment of $\leq 1 / 10$ on a visual analogue scale (VAS) as an absolute requirement for remission. While such a low VAS may be achievable in patients in the controlled clinical trials in which the ACR/EULAR criteria were validated, it is becoming increasingly apparent that patient VAS can be influenced by non-RA factors including osteoarthritis and other medical comorbidities. Indeed, several groups now suggest that the VAS threshold in ACR/EULAR Boolean remission may be overly strict and underdiagnose remission when used in 'real-world' clinical practice. ${ }^{3-5}$ In this regard, it is interesting to note that in the original publication of the ACR/EULAR remission criteria, the consensus survey of expert opinion centred on a higher patient VAS threshold of 2.2/10 when all other parameters were consistent with remission.

Third, ACR/EULAR remission criteria neglect measures of synovitis by imaging modalities such as ultrasound (US) and MRI-arguably a more stringent measure of joint inflammation than clinical examination alone. Although ACR/EULAR remission has been shown to correlate with lower levels of US synovitis compared with DAS28, ${ }^{6}$ we and other groups have demonstrated that power Doppler synovitis can still be detected in patients who satisfy ACR/EULAR remission criteria, with a prevalence as high as $60 \% .^{78}$

Fourth, ACR/EULAR remission appears to afford no clear advantage over DAS28-based definitions when applied to the identification of patients in remission who can successfully reduce or even stop their disease-modifying antirheumatic drug (DMARD) therapy. In the Reduction of Therapy in patients with Rheumatoid arthritis in Ongoing remission (RETRO) study, Boolean ACR/EULAR remission at baseline did not predict sustained DMARD-free remission, ${ }^{9}$ whereas both autoantibody status and serum cytokine levels provided added value in identifying patients whose disease flared following DMARD withdrawal. ${ }^{10}$

In conclusion, while we acknowledge and support the vital work to reach an international consensus on defining RA remission, this is by no means a fait accompli. ACR/EULAR remission does not always equate with absence of disease and is not necessarily the optimal definition for application in clinical practice, particularly in non-research settings. There is an urgent need for robust and practical biomarkers that can better measure RA remission which, once discovered and validated, could be used to improve future definitions of RA remission.

\section{Kenneth F Baker, Arthur G Pratt, Ben Thompson, John D Isaacs}

Musculoskeletal Research Group, NIHR Newcastle Biomedical Research Centre, Newcastle University and Newcastle upon Tyne Hospitals NHS Foundation Trust, Newcastle upon Tyne, UK

Correspondence to Dr Kenneth F Baker, Musculoskeletal Research Group, NIHR Newcastle Biomedical Research Centre, Newcastle University and Newcastle upon Tyne Hospitals NHS Foundation Trust, 4th Floor Catherine, Cookson Building, Framlington Place, Newcastle upon Tyne NE2 4HH, UK; k.f.baker@ncl.ac.uk

\section{Competing interests None.}

Provenance and peer review Not commissioned; internally peer reviewed.

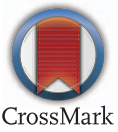

To cite Baker KF, Pratt AG, Thompson B, et al. Ann Rheum Dis 2017;76:e12.

Accepted 9 November 2016

Published Online First 1 December 2016

Ann Rheum Dis 2017;76:e12. doi:10.1136/annrheumdis-2016-210797

\section{REFERENCES}

1 Boers M. Let's stop fooling ourselves. In RA, only ACR/EULAR criteria define remission and equate with absence of disease! Ann Rheum Dis 2016;75:e68

2 Felson DT, Smolen JS, Wells G, et al. American College of Rheumatology/European League Against Rheumatism provisional definition of remission in rheumatoid arthritis for clinical trials. Arthritis Rheum 2011;63:573-86.

3 Vermeer $M$, Kuper $H H$, van der Bijl AE, et al. The provisional ACR/EULAR definition of remission in RA: a comment on the patient global assessment criterion. Rheumatology (Oxford) 2012;51:1076-80.

4 Masri KR, Shaver TS, Shahouri SH, et al. Validity and reliability problems with patient global as a component of the ACR/EULAR remission criteria as used in clinical practice. J Rheumatol 2012;39:1139-45.

5 Studenic P, Smolen JS, Aletaha D. Near misses of ACR/EULAR criteria for remission: effects of patient global assessment in Boolean and index-based definitions. Ann Rheum Dis 2012;71:1702-5.

6 Sakellariou G, Scirè CA, Verstappen SM, et al. In patients with early rheumatoid arthritis, the new ACR/EULAR definition of remission identifies patients with persistent absence of functional disability and suppression of ultrasonographic synovitis. Ann Rheum Dis 2013;72:245-9.

7 Horton SC, Tan AL, Freeston JE, et al. Discordance between the predictors of clinical and imaging remission in patients with early rheumatoid arthritis in clinical practice: implications for the use of ultrasound within a treatment-to-target strategy. Rheumatology (Oxford) 2016;55:1177-87.

8 Baker KF, Thompson B, Lendrem DW, et al. Ultrasound measures of synovitis are independent of clinical parameters in the setting of rheumatoid arthritis remission: a cross-sectional analysis. Ann Rheum Dis 2016;75(Suppl 2):1228.

9 Haschka J, Englbrecht M, Hueber AJ, et al. Relapse rates in patients with rheumatoid arthritis in stable remission tapering or stopping antirheumatic therapy: interim results from the prospective randomised controlled RETRO study. Ann Rheum Dis 2016;75:45-51.

10 Rech J, Hueber AJ, Finzel $\mathrm{S}$, et al. Prediction of disease relapses by multibiomarker disease activity and autoantibody status in patients with rheumatoid arthritis on tapering DMARD treatment. Ann Rheum Dis 2016;75:1637-44. 